\title{
Bounds on diffuse and point source fluxes of ultra-high energy neutrinos with the Pierre Auger Observatory
}

\author{
Francisco Pedreira ${ }^{* a}$ for the Pierre Auger Collaboration ${ }^{b \dagger}$ \\ a IGFAE, Santiago de Compostela, Spain \\ ${ }^{b}$ Observatorio Pierre Auger, Av. San Martín Norte 304, 5613 Malargüe, Argentina \\ E-mail: auger_spokespersons@fnal.gov \\ Full author list: http://www.auger.org/archive/authors_icrc_2019.html
}

\begin{abstract}
Neutrinos with energies above $100 \mathrm{PeV}$ are detectable with the Surface Detector array (SD) of the Pierre Auger Observatory. Identification is efficiently done for neutrinos of all flavors interacting in the atmosphere at large zenith angles (downward-going DG), as well as for Earth-skimming (ES) tau neutrinos. No neutrino candidates were found up to 31 August 2018, and this allowed us to put upper bounds on their flux that constrain several models of cosmic-ray and neutrino production at EeV energies. With the SD of Auger we can also search for neutrinos from point-like sources, monitoring a large fraction of the sky (from $\sim-80^{\circ}$ to $\sim+60^{\circ}$ ) in equatorial declination with peak sensitivities at declinations around $-53^{\circ}$ and $+55^{\circ}$, unmatched in the northern hemisphere. An excellent sensitivity can also be obtained in the case of transient sources lasting of order an hour or less if they occur when the source is in the field of view of the ES or DG channels.
\end{abstract}

36th International Cosmic Ray Conference - ICRC2019

24 July - 1 August, 2019

Madison, Wisconsin, USA

\footnotetext{
* Speaker.

${ }^{\dagger}$ for collaboration list see PoS(ICRC2019)1177
} 


\section{Introduction}

In this work we report on the search for ultra-high energy (UHE) neutrinos in data taken with the SD of the Pierre Auger Observatory. A blind scan of data from 1 January 2004 up to 31 August 2018 has yielded no neutrino candidates. This corresponds to $\sim 14.7$ years of data taken or to $\sim 9.7$ equivalent years of operation of a complete SD (the array was not fully deployed until 2008) representing an increase of 5.2 years of data taken over previous searches [1]. The non-observation of neutrino candidates allows us to place stringent constraints on the diffuse flux of UHE neutrinos with relevant implications for the origin of the ultra-high energy cosmic rays (UHECRs). Here we report upper limits to the diffuse flux of UHE neutrinos [2] and also corresponding limits to the neutrino flux from point-like sources as a function of declination [3].

\section{The neutrino search with the Pierre Auger Observatory}

The Pierre Auger Observatory is located in the province of Mendoza, Argentina, at a mean altitude of $1400 \mathrm{~m}$ above sea level. It has been running and taking data since 2004, and although it was primarily designed to measure extensive air showers (EAS) induced by UHECRs, the Observatory can also identify UHE neutrinos using its SD. This is an array of 1660 water-Cherenkov detectors ("stations"), each SD station containing 12 tonnes of water and located $1.5 \mathrm{~km}$ from its neighbors arranged in a hexagonal pattern.

There are two different ways to detect neutrinos with the SD. Firstly, neutrinos of all flavors can undergo charged current (CC) or neutral current (NC) interactions in the atmosphere and induce a "downward-going" shower that can be detected. Secondly, tau neutrinos $\left(v_{\tau}\right)$ can produce in the Earth crust a tau lepton (through CC interactions) that exits the Earth surface and decays in the atmosphere inducing an "Earth-skimming" upward-going shower. Neutrino-induced showers must be identified in Auger data against the background of showers initiated by UHECRs. The identification is based on a simple idea: protons, heavier nuclei and photons interact shortly after entering the atmosphere, while neutrinos can initiate showers deep in the atmosphere. At large zenith angles, the atmosphere is thick enough that the electromagnetic component of showers initiated by nucleonic cosmic rays gets absorbed, and the shower front at ground level is mainly composed of muons producing signals in the SD stations that have characteristic large peaks associated to individual muons which are spread over short time intervals. On the other hand, showers induced by neutrinos deep in the atmosphere have a considerable electromagnetic component at the ground, in several of the triggered SD stations producing signals that are spread over time.

The search strategy consists in selecting showers that arrive at the SD array in the inclined directions (with zenith angle between $60^{\circ}<\theta<90^{\circ}$ for DG and $90^{\circ}<\theta<95^{\circ}$ for ES) and identifying those that show a broad time structure in the signals detected for the SD stations. In the following we briefly explain the main steps involved in the selection algorithm. The first step is the selection of inclined events. For geometrical reasons, in inclined events the pattern of the triggered SD stations typically exhibits an elliptical shape on the ground. These patterns can be characterized by a length $L$ (major axis) and a width $W$ (minor axis) and inclined events typically exhibit large values of $L / W$ [4]. The different set of cuts applied to select DG and ES events is detailed in [2]. 
The second step is identifying those inclined events that interact deep in the atmosphere. The main observable used to discriminate broad from narrow shower fronts is the Area-over-Peak ${ }^{1}$ (AoP) [1]. Inclined background showers of hadronic origin exhibit AoP values close to one, in contrast with neutrino-induced showers where the values of AoP are typically larger.

To select ES $v_{\tau}$, we use the AoP averaged over all the triggered stations $(\langle\mathrm{AoP}\rangle)$. For DG showers, we construct a multivariate analysis combining several observables that carry information on the time spread of the signals in the SD stations. For optimization purposes the DG category of events is subdivided into two sets for Low (DGL) and High (DGH) zenith angles, between $60^{\circ}<\theta<75^{\circ}$ and $75^{\circ}<\theta<90^{\circ}$ [1], respectively. Full details can be found in [2].

A search for ES and DG neutrino-induced showers was performed in the Observatory data from 1 January 2004 when data taking started, up to 31 August 2018. No neutrino candidates were identified. In Fig. 1 (left) we show the distribution of $\langle\mathrm{AoP}\rangle$ for inclined events in the ES sample in the whole data period compared to that expected in Monte Carlo simulations of $v_{\tau}$-induced ES showers, along with the optimized value of the cut $(\langle\mathrm{AoP}\rangle=1.83)$ above which an event would be regarded as a neutrino candidate [2]. After the inclined selection and the neutrino identification criteria $\sim 95 \%$ of the simulated neutrinos that induce triggers are identified. This proves that the Pierre Auger Observatory is highly efficient as a neutrino detector. The Fisher distribution for inclined events in the DGH sample in the whole data period with $N_{\text {stations }} \geq 12$ compared to that expected in Monte Carlo simulations is also shown in Fig. 1 (right).

\section{Exposure}

The non-observation of neutrino candidates let us calculate an upper limit to the diffuse flux of UHE neutrinos. For this purpose the exposure of the SD of Auger needs to be obtained for
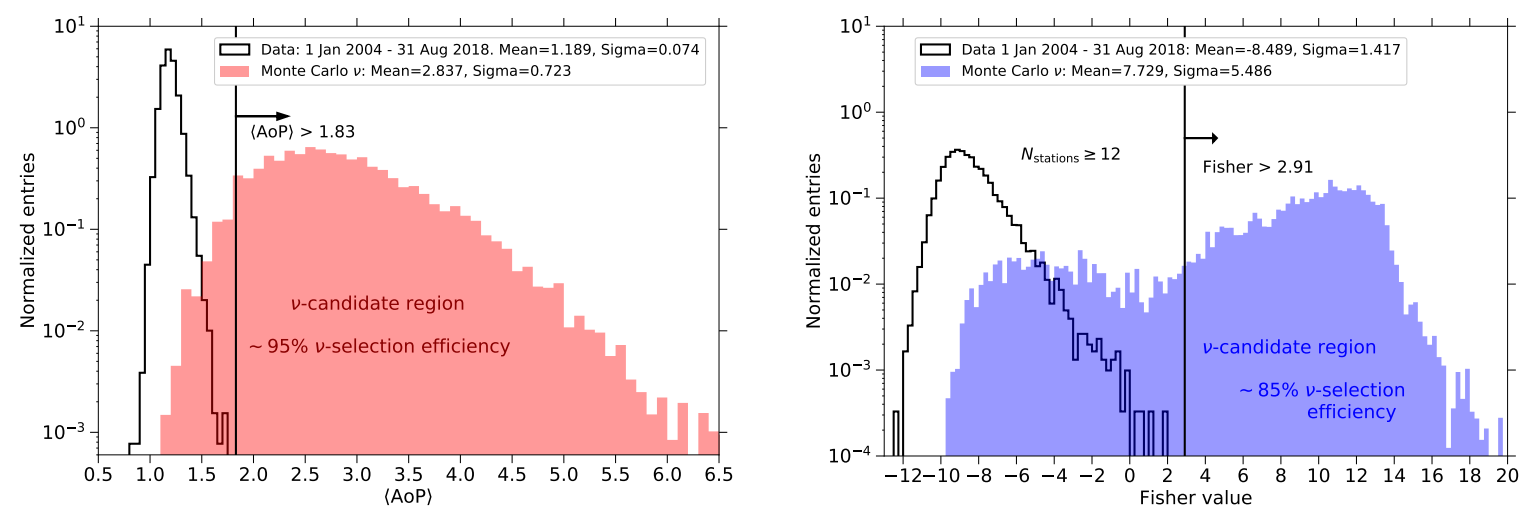

Figure 1: Left: Distribution of $\langle A o P\rangle$ after the Earth-Skimming inclined selection. Right: Distribution of the Fisher variable after the DGH inclined event selection for events with a number of triggered stations larger than 11. The filled red and blue histograms are the distributions of $\langle\mathrm{AoP}\rangle$ and Fisher value in Monte Carlo (MC) simulations of $v$. Black histograms refer to the corresponding selection for data (25904 inclined events in left, 12139 inclined events in right).

\footnotetext{
${ }^{1}$ The Area-over-Peak is defined as the ratio of the integral of the time trace to its peak value normalized to the average signal produced by a single vertical muon.
} 
the period unblinded using Monte Carlo simulations of neutrino-induced showers, as well as the same selection and identification criteria applied to the data. The identification efficiencies for each channel are shown in [4].

For downward-going neutrinos the detection efficiency, $\varepsilon_{i, c}$, depends mainly on neutrino flavor $i=v_{e}, v_{\mu}, v_{\tau}$, the type of interaction ( $c=\mathrm{CC}, \mathrm{NC}$ ), neutrino energy $E_{v}$, zenith $\theta$ and azimuth $\varphi$ angles, the point of impact of the shower core on the ground, and the depth at which the neutrino interacts. In the $v_{\tau}$ Earth-skimming channel the efficiency $\varepsilon_{\mathrm{ES}}$ depends on the energy of the emerging $\tau$ leptons $E_{\tau}$, the position of the signal pattern on the ground, on the zenith $\theta$ and azimuth $\varphi$ angles, and on the altitude of the decay point of the $\tau$ above the ground. The total exposure $\mathscr{E}_{\text {tot }}$ is obtained assuming a flavor mixture of $v_{e}: v_{\mu}: v_{\tau}=1: 1: 1$. Full details can be found in [2].

\section{Limits to diffuse fluxes}

The total exposure $\mathscr{E}_{\text {tot }}$ folded with a single-flavor flux of UHE neutrinos per unit energy, area $A$, solid angle $\Omega$ and time, and integrated in energy gives the expected number of events for that flux:

$$
N_{\mathrm{evt}}=\int_{E_{v}} \mathscr{E}\left(E_{v}\right) \phi\left(E_{v}\right) \mathrm{d} E_{v}
$$

Assuming a differential neutrino flux $\phi=k \cdot E_{v}^{-2}$, an upper limit to the value of $k$ at $90 \%$ C.L. is obtained as

$$
k_{90}=\frac{2.39}{\int_{E_{v}} E_{v}^{-2} \mathscr{E}_{\text {tot }}\left(E_{v}\right) \mathrm{d} E_{v}},
$$

where 2.39 is the Feldman-Cousins factor for non-observation of events in the absence of expected background accounting for systematic uncertainties [1]. The single-flavor 90\% C.L. integrated limit is $k_{90}<4.4 \times 10^{-9} \mathrm{GeV} \mathrm{cm}^{-2} \mathrm{~s}^{-1} \mathrm{sr}^{-1}$. It mostly applies in the energy interval $10^{17} \mathrm{eV}-$ $2.5 \times 10^{19} \mathrm{eV}$ for which $\sim 90 \%$ of the total event rate is expected in the case of a $E_{v}^{-2}$ spectral flux. The integrated limit represents the value of the normalization of a $E_{v}^{-2}$ differential neutrino flux needed to predict $\sim 2.39$ expected events. For such a spectral shape tau neutrinos contribute up to $\sim 86 \%$ of the total event rate, and in particular ES neutrinos dominate the rate of $v_{\tau}$ events over the downward-going $v_{\tau}$. The contribution of $v_{e}$ and $v_{\mu}$ together is smaller than $15 \%$ in this case.

The denominator of Eq. (4.2) can also be integrated in bins of neutrino energy of width $\Delta E_{v}$, and a limit $\hat{k}_{90}$ can be obtained in each energy bin. This is shown in Fig. 2 (left) for logarithmic energy intervals $\Delta \log _{10} E_{v}=0.5$. The differential limit is an effective way of characterizing the energy dependence of the sensitivity of a neutrino experiment. It can be seen in Fig. 2 that the best sensitivity is achieved for energies around $1 \mathrm{EeV}$ in Auger.

\section{Constraints on the origin of UHECRs}

Employing the upper limit obtained with the Observatory, we can constrain several models of neutrino production in interactions of UHECRs with the cosmic microwave background, often referred to as cosmogenic neutrino models. For example, scenarios assuming sources that accelerate only protons and which have a strong evolution with $z$, similar to Fanaroff-Riley type II (FRII) radio-loud active galactic nuclei (AGN) [5], are strongly constrained (probability of observing 0 neutrinos is $\sim 2.7 \times 10^{-3}$ ) by the Auger results at more than $90 \%$ C.L. 
To explore the constraining power of the Auger upper limits to $\mathrm{EeV}$ neutrinos, we performed an extensive scanning of the evolution function of the sources with redshift, $\Psi(z) \propto(1+z)^{m}$ up to $z_{\max }$ (maximal redshift at which UHECRs are accelerated). For each pair of $m$ and $z_{\max }$, the cosmogenic flux was calculated, and the expected number of neutrino events in Auger was obtained. Models predicting more than 2.39 neutrinos are disfavored at $>90 \%$ C.L. The resultant exclusion plot is shown in Fig. 2 (right) with the contours representing the $68 \%$ and $90 \%$ C.L. exclusion limits, respectively. The non-observation of neutrino candidates in the Observatory data allows us to exclude a significant region of the parameter space $\left(m, z_{\max }\right)$ [4].

\section{Sensitivity of the Observatory to point-like neutrino sources}

As the Pierre Auger Observatory has a good angular resolution (typically better than $2.5^{\circ}$ ) [10], we can also obtain a limit to point-like sources of UHE $v$. Each neutrino search channel, ES, DGH and DGL, corresponds to a given range of zenith angles, and the three combined cover between $\theta=60^{\circ}$ and $95^{\circ}$. The neutrino identification efficiency is different in each channel, making the sensitivity of the Observatory dependent on the direction in the sky where the search is performed. The sensitivity in each direction can be quantified in terms of the effective area $\mathscr{A}_{i}\left(E_{V}\right)$, to neutrinos of flavor $i=v_{e}, v_{\mu}, v_{\tau}$ and energy $E_{v}$, defined such that $\mathscr{A}_{i}$ multiplied by the spectral flux of flavor $i$ from a point source gives the energy spectrum of the instantaneous rate of detected events. The instantaneous effective area for the ES, DGH and DGL neutrinos as a function of neutrino energy is displayed in Fig. 3 (left). The Pierre Auger Observatory has optimal effective area in the EeV
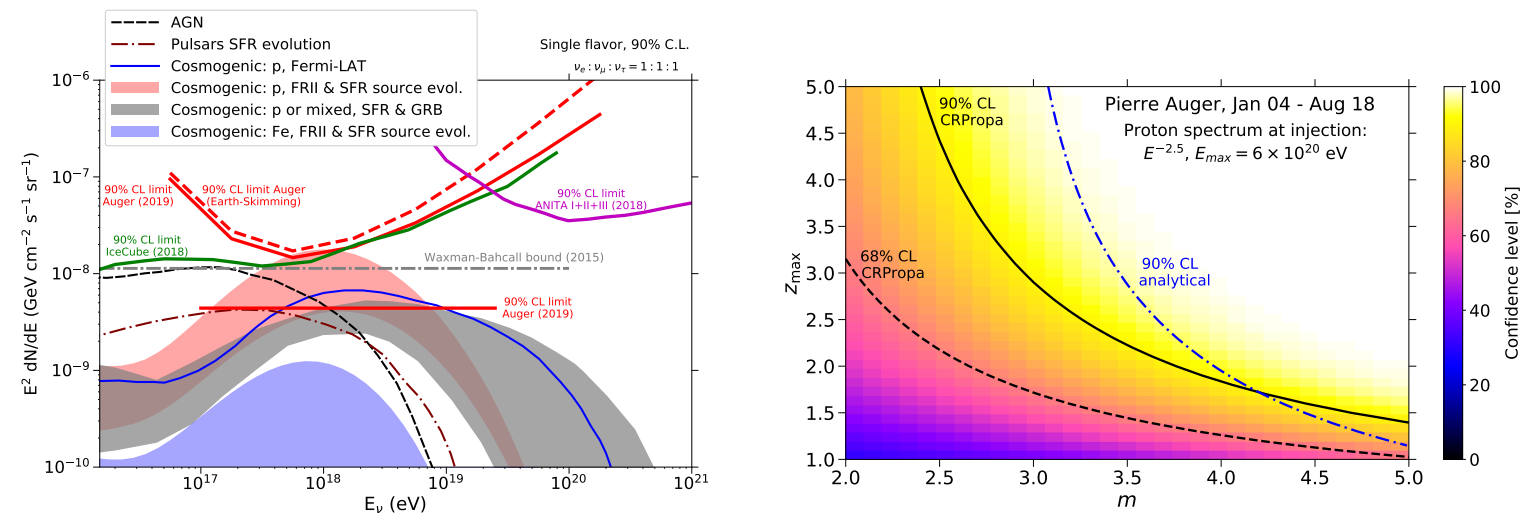

Figure 2: Left: Pierre Auger Observatory upper limit (90\% C.L.) to the normalization $k$ of the diffuse flux of UHE neutrinos $\phi_{v}=k E_{v}^{-2}$ as given in text (solid straight red line). Also plotted are the upper limits to the normalization of the diffuse flux (differential limits) when integrating the denominator of Eq. (4.2) in bins of width 0.5 in $\log _{10} E_{v}$ (solid red line - Auger all channels and flavors; dashed red line - Auger Earth-skimming $v_{\tau}$ only). Similar limits from ANITA I+II+III [6] and IceCube [7] are displayed along with predictions for several neutrino models. All limits and fluxes are converted to a single flavor. Right: Constraints on UHECR source evolution models parameterized as $\psi(z) \propto(1+z)^{m}$ for sources distributed homogeneously up to a maximum redshift $z_{\max }$ and emitting protons following a power-law $\mathrm{d} N / \mathrm{d} E \propto E^{-2.5}$ up to $E=6 \cdot 10^{20} \mathrm{eV}$. A proton-only flux is matched to the Auger spectrum at $7 \times 10^{18} \mathrm{eV}$. The cosmogenic neutrino fluxes for each combination of $m$ and $z_{\max }$ were calculated with the MC propagation code CRPropa [8]. See [2] for further references and details on these plots. 
energy range beyond the published effective area of IceCube and, for favorable source positions as seen from the SD, the effective area of the Pierre Auger Observatory is significantly larger.

As neutrinos at the Pierre Auger Observatory are searched for $\theta$ between $90^{\circ}$ and $95^{\circ}$ in the ES analysis, and between $60^{\circ}$ and $90^{\circ}$ in the DG analysis, at each instant, neutrinos can be effectively detected only from a specific region of the sky corresponding to this zenith angle range. A pointlike source at a declination $\delta$, right ascension $\alpha$ (equatorial coordinates) and a local sidereal time $t$, is seen from the latitude of the Observatory $\left(\lambda=-35.2^{\circ}\right)$ with a time-dependent zenith angle $\theta(t)$ given by $\cos \theta(t)=\sin \lambda \sin \delta+\cos \lambda \cos \delta \sin (2 \pi t / T-\alpha)$ where $T$ is the duration of one sidereal day. At any given instant, the field of view (FoV) of the Observatory for neutrino searches is limited by the selection imposed on $\theta$. In fact, the three channels ES, DGH and DGL correspond to different FoV. This is shown in Fig. 3 (right) where the corresponding FoV bands are plotted in equatorial coordinates as a function of $\alpha-t_{\mathrm{GS}}$, where $t_{\mathrm{GS}}=2 \pi t / T+\ell$ is the Greenwich Sidereal Time (GST) converted to angle and $\ell$ is the mean longitude of the Observatory. For any given $\alpha$ the instantaneous declination range for neutrino search at 00:00 GST $\left(t_{\mathrm{GS}}=0\right)$ can be read from the plot at a value $\alpha$ of the abscissa. At any other $t_{\mathrm{GS}}$ the corresponding declination range is simply read from Fig. 3 at $\alpha-t_{\mathrm{GS}}$.

The SD of the Pierre Auger Observatory is sensitive to point-like sources of neutrinos over a broad declination range between $\delta \sim-85^{\circ}$ and $\delta \sim 60^{\circ}$ (see Fig. 3). In particular ES showers can be efficiently identified between $\delta \sim-55^{\circ}$ and $\delta \sim 60^{\circ}$ (red band), for DGH showers between $\delta \sim-70^{\circ}$ and $\delta \sim 55^{\circ}$ (blue band), and for DGL between $\delta \sim-85^{\circ}$ and $\delta \sim 40^{\circ}$ (green band). In the case of a steady flux we can calculate the expected energy distribution of the detected events by multiplying the exposure $\mathscr{E}(\delta)$ to a point-like source of UHE neutrinos by the spectral flux. The exposure depends on neutrino energy $E_{v}$ and on the declination $\delta$ of the source and is obtained by integrating the effective area $\mathscr{A}$ over a given time interval. For a given source position $\delta$, the effective area $\mathscr{A}$ is dependent on $\theta$ which changes with time.
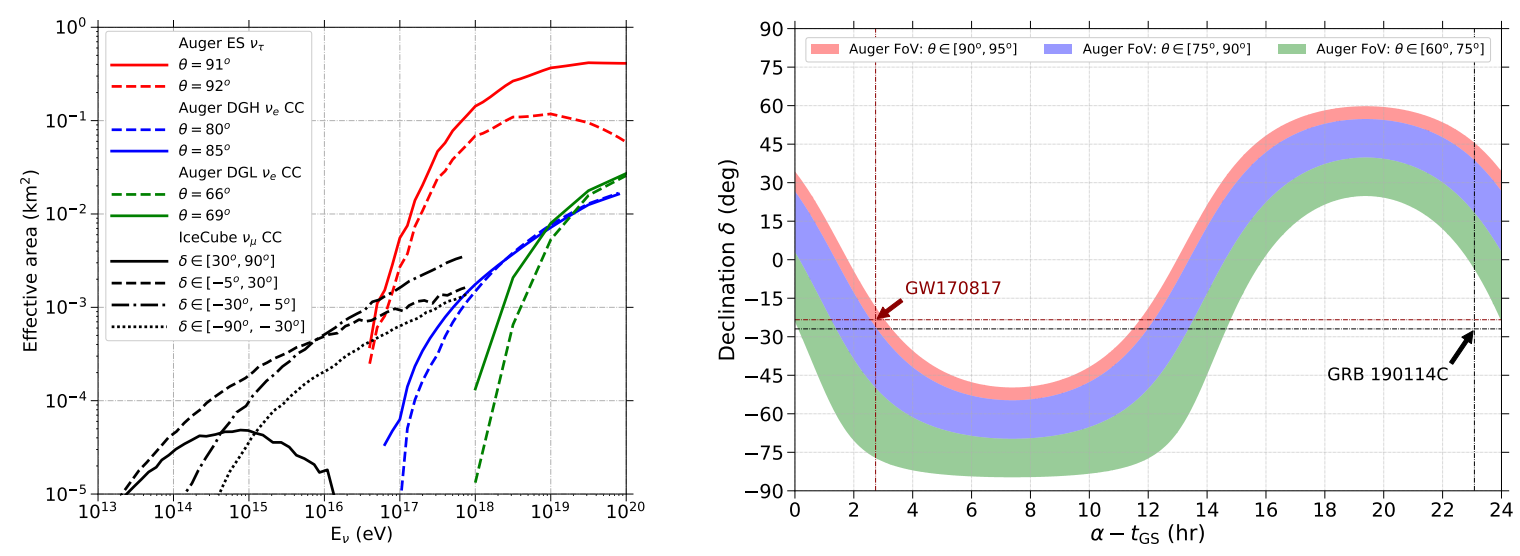

Figure 3: Left panel: Instantaneous effective areas for all the channels as a function of neutrino energy for selected zenith angles as labeled, compared to those of IceCube [11]. Right panel: Instantaneous FoV of the Pierre Auger Observatory for ES, DGH and DGL neutrinos as a function of the hour angle (see text). Two source examples are shown: GW170817 visible in Auger in the ES at the time of emission [12], and GRB 190114C [13] not visible in Auger in the inclined directions at the time of the burst. 
The directional exposure obtained daily for the ES, DGH and DGL selections at fixed energies, averaged over the period between 1 May 2008 and 31 August 2018 (excluding the intervals over which the array was unstable) is shown in Fig. 4 (left) as a function of the source declination. Two sets of curves for each selection group have been combined in Fig. 4 for $10^{18} \mathrm{eV}$ and $10^{19.5} \mathrm{eV}$ to illustrate that the relative weight of the channels depends strongly on the energy. The position of the source relative to the Observatory during the search period plays a crucial role in the case of searches for short time intervals. When the source lies just below the horizon, at a zenith angle $\theta \sim 90^{\circ}-93^{\circ}$, the effective area is maximal and the integrated exposure is competitive with dedicated $v$ experiments such as IceCube and ANTARES.

\section{Limits for steady sources of UHE neutrinos}

As stated in section 4, a blind search for UHE neutrinos in the data period analyzed has yielded no candidate neutrino events in the ES, DGH, and DGL analyses. Under the same conditions that we consider in section 4, a bound on $k_{\mathrm{PS}}(\boldsymbol{\delta})$ can be obtained separately for the ES, DGH, and DGL channels. In each of the DG channels the contributions from different flavors having both NC and $\mathrm{CC}$ are combined in the equal flavor assumption. In the calculation of the limits, the dependence of the neutrino detection efficiency on the zenith angle and its change with time as the source transits in the Observatory field of view are taken into account.

The limits are shown in Fig. 4 (right) as a function of declination in comparison to those obtained by IceCube [11] and ANTARES [14]. It must be emphasized that the energy ranges where the three experiments are sensitive, are different and complementary. The limits reported by ANTARES and IceCube apply to energies below the energy range of the search of neutrinos with Auger.
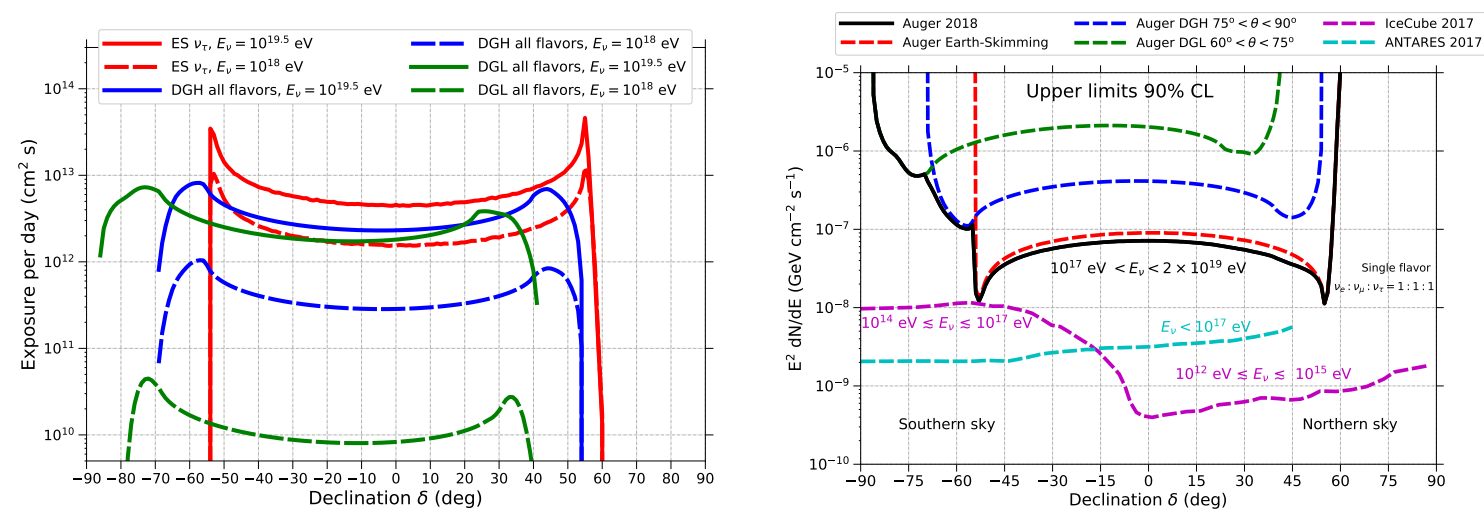

Figure 4: Left panel: Average exposure per day at $E_{v}=10^{18} \mathrm{eV}$ and $3 \times 10^{19} \mathrm{eV}$ as a function of declination $\delta$ for the ES, DGH and DGL channels. Right panel: Pierre Auger Observatory upper limits (1 Jan 2004 31 Aug 2018) at $90 \%$ C.L. on the normalization $k^{\mathrm{PS}}$ of a single flavor point-like flux of UHE neutrinos $\mathrm{d} N / \mathrm{d} E_{v}=k^{\mathrm{PS}} E_{v}^{-2}$ as a function of the source declination $\delta$. The limits for IceCube (2008 - 2015) [11] and ANTARES (2007 - 2015) [14] are also shown. Note the different energy ranges for each observatory. 


\section{Discussion and conclusions}

With the Pierre Auger Observatory we can detect UHE neutrinos from a large fraction of the sky, from very close to the South Celestial Pole to declination values up to $\delta \sim 60^{\circ}$. For a steady source in this range there is always a time window during a sidereal day in which the source would be in the field-of-view of the ES, DGH or DGL channels.

No neutrino candidates have been identified in Auger data until 31 Aug 2018. The upper limits to the diffuse flux of UHE $v$ obtained with the Pierre Auger Observatory are competitive at energies around $10^{18} \mathrm{eV}$. The SD of the Pierre Auger Observatory has an unmatched sensitivity to potential sources of EeV neutrinos in the Northern terrestrial hemisphere. This region in the sky cannot be searched for in the EeV energy range by experiments such as IceCube because of the opacity of the Earth to neutrinos in those directions when seen from the South Pole.

The sensitivity of the SD of the Pierre Auger Observatory to transient sources of UHE neutrinos is crucially dependent on the efficiency of the detection during the time interval of the transient. Thus, depending on the inclination of the event in the local coordinate system of the Observatory, the sensitivity can exceed by far that of other dedicated neutrino detectors. For instance, at about $10^{18} \mathrm{eV}$, the Pierre Auger Observatory's effective area is maximal for sources slightly below the horizon, and therefore its sensitivity to transients from these directions is larger than that of IceCube by more than an order of magnitude. This is particularly interesting because the location of the GW170817 event, the first known binary neutron star merger, was slightly below the horizon at the time around the merger as reported in [12] and seen in Fig. 3.

The Pierre Auger Observatory is placed in an excellent position to contribute to the recently started era of multi-messenger astronomy, which is likely to bring new exciting discoveries in the near future, by looking for neutrinos in the $\mathrm{EeV}$ range in correlation with the detection of gamma rays or gravitational waves.

\section{References}

[1] A. Aab et al. (Pierre Auger Collab.), Phys. Rev. D 91, 092008 (2015).

[2] A. Aab et al. (Pierre Auger Collab.), submitted to JCAP (2019) arXiv:1906.07422.

[3] A. Aab et al. (Pierre Auger Collab.), submitted to JCAP (2019) arXiv:1906.07419.

[4] E. Zas for the Pierre Auger Collab., PoS(ICRC2017) 972.

[5] K.-H. Kampert, and M. Unger, Astropart. Phys. 35, 660 (2012).

[6] P. W. Gorham et al. (ANITA Collab.), Phys. Rev. D 98, 022001 (2018).

[7] M. G. Aartsen et al. (IceCube Collab.), Phys. Rev. D 98, 062003 (2018).

[8] R. Alves Batista et al., JCAP 05, 038 (2016).

[9] A. Ishihara and S. Yoshida, Phys. Rev. D 85, 063002 (2012).

[10] A. Aab et al. (Pierre Auger Collab.), JCAP 08, 019 (2014).

[11] M. G. Aartsen et al. (IceCube Collab.), Astrophys. J. 835, 151 (2017).

[12] A. Albert et al. (ANTARES, IceCube, Pierre Auger \& LIGO/Virgo Collabs.), Astrophys. J. Lett. 850, L35 (2017).

[13] http://tevcat.uchicago.edu/?mode=1;id=324

[14] A. Albert et al. (ANTARES Collab.), Phys. Rev. D 96, 082001 (2017). 